PROCEEDINGS OF THE

AMERICAN MATHEMATICAL SOCIETY

Volume 130, Number 10, Pages 2905-2909

S 0002-9939(02)06644-3

Article electronically published on May 8, 2002

\title{
A GENERALIZATION OF LOMONOSOV'S INEQUALITY AND ITS APPLICATIONS TO INVARIANT SUBSPACES
}

\author{
SHAMIM I. ANSARI
}

(Communicated by David R. Larson)

\begin{abstract}
In this article we generalize Victor Lomonosov's famous inequality so as to be applicable to a wider class of functions. Then using it we prove that the adjoint of an algebra with a compactness property which is weaker than the PS property, employed by Victor Lomonosov, has nontrivial invariant subspaces.
\end{abstract}

\section{INTRODUCTION}

In this article the phrase "weakly closed" stands for "closed relative to the weak operator topology". The symbol " $X$ " represents a Banach space over either the field of real numbers or the complex numbers, " $X^{*}$ " denotes the dual of $X$, and $L(X)$ the algebra of all continuous linear operators from $X$ to itself. In his 1991 famous work on the extension of Burnside's theorem Victor Lomonosov proved an inequality for the adjoints of elements of a weakly closed proper subalgebra of $L(X)$. As an application of this inequality he proved that the adjoint of a weakly closed proper subalgebra with the PS (see [L2]) property has nontrivial invariant subspaces. This raised the problem, whether each operator is contained in a proper weakly closed subalgebra of the whole algebra with the PS property. It was then a natural question to ask if the commutant of each Hilbert space operator has the PS property. Don Hadwin (1995) answered the latter question in the negative. Here we generalise Victor Lomonosov's inequality (Theorem 1), using his own arguments, so as to be satisfied by a larger class of functions. Then we use this inequality to prove the existence of invariant subspaces for the adjoint of a weakly closed proper subalgebra with a property weaker than the PS property. We call this weaker property the LPS (Lomonosov-Pearcy-Salinas) property. Don Hadwin's example has LPS. In fact if $X$ is reflexive and separable, then it can be renormed so that any proper unital weakly closed subalgebra of $L(X)$ with a nontrivial closed invariant subspace has this property (see Proposition 1 ). In this sense LPS is a necessary and sufficient condition for a proper unital weakly closed subalgebra of $L(X)$ to have nontrivial closed invariant subspaces. The references given here include only those papers that are employed here. In fact, there are some very interesting articles in the literature related to V. Lomonosov's inequality.

Received by the editors December 15, 2000.

2000 Mathematics Subject Classification. Primary 47A15.

(C)2002 American Mathematical Society 
Notation. The letter " $K$ " stands for a $w^{*}$ compact subset of $X^{*} . C(K)$ (respectively, $C\left(K, X^{*}\right)$ ) denotes the space of all scalar valued (respectively, $X^{*}$ valued) $w^{*}$-norm continous functions on $K . W^{*}\left(K, X^{*}\right)$ represents the space of all $X^{*}$ valued $w^{*}-w^{*}$ continuous functions on $K$. The norm on all the spaces mentioned will be the sup norm on $K$ and for $G \in W^{*}\left(K, X^{*}\right),\left\||| G\left|\left\|_{K}=\right\|\right||G|\right\| \mid=d\left(G, C\left(K, X^{*}\right)\right)$. $\mathcal{R}$ is a weakly closed proper subalgebra of the space of all continuous linear operators from $X$ to $X$ and $\mathcal{R}^{*}$ the adjoint of $\mathcal{R}$, that is, $\mathcal{R}^{*}=\left\{A^{*} \mid A \in \mathcal{R}\right\}$. If $A^{*} \in \mathcal{R}^{*}$, the restriction $\left.A^{*}\right|_{K} \in W^{*}\left(K, X^{*}\right) .\left.A^{*}\right|_{K}$ will simply be denoted $A^{*}$. If $f \in C(K)$ and $A \in \mathcal{R}$, then $f A^{*} \in W^{*}\left(K, X^{*}\right)$ is defined by $\left(f A^{*}\right)\left(x^{*}\right)=f\left(x^{*}\right) A^{*}\left(x^{*}\right)$ for all $x^{*} \in K$. The vector space of elements of the form $\sum_{i=1}^{n} f_{i} A_{i}^{*}$ with $f_{i} \in C(K)$ and $A_{i}^{*} \in \mathcal{R}^{*}$ will be denoted $F\left(\mathcal{R}^{*}(K)\right)$ and its norm closure in $W^{*}\left(K, X^{*}\right)$ by $\overline{F\left(\mathcal{R}^{*}(K)\right)}$.

Theorem 1 (A Generalization of Lomonosov's Inequality). Let $\mathcal{R}$ be a weakly closed proper subalgebra of $L(X)$. Then, either $\mathcal{R}$ has nontrivial invariant subspaces or for any closed ball $K$ of positive radius there exist $x_{0}^{* *} \in X^{* *}, x_{0}^{*} \in K$, $x_{0}^{* *} \neq 0, x_{0}^{*} \neq 0$ such that for all $G \in \overline{F\left(\mathcal{R}^{*}(K)\right)}$,

$$
\left|x_{0}^{* *}\left(G x_{0}^{*}\right)\right| \leq\||| G \mid\| .
$$

Proof. Suppose $\mathcal{R}$ has no nontrivial invariant subspaces. Let $M$ be the norm closed linear span of $\overline{F\left(\mathcal{R}^{*}(K)\right)} \cup C\left(K, X^{*}\right)$ in $W^{*}\left(K, X^{*}\right)$. Using the fact that $\mathcal{R}$ is weakly closed and is not the whole algebra $L(X)$ and employing V. Lomonosov's work readers can verify that $\overline{F\left(\mathcal{R}^{*}(K)\right)}$ is not norm dense in $M$ and that there is a functional $\phi \in M^{*}$ such that $\phi\left(\overline{F\left(\mathcal{R}^{*}(K)\right)}\right)=0$ and for all elements $C \in$ $C\left(K, X^{*}\right), \phi(C)=x_{1}\left(C x_{0}^{*}\right)$ for some $x_{1} \in X^{* *}$ and some $x_{0}^{*} \in K$ with $x_{1} \neq$ $0, x_{0}^{*} \neq 0$. Consider the continuous linear functional $\delta$ on $W^{*}\left(K, X^{*}\right)$ defined by $\delta(G)=x_{1}\left(G x_{0}^{*}\right)$. Then $\|\delta\| \leq\left\|x_{1}\right\|$. For any $G \in \overline{F\left(\mathcal{R}^{*}(K)\right)}$ we have $0=\phi(G)=$ $(\phi-\delta)(G)+\delta(G)$. Note that for any bounded linear functional $f$ on a Banach space and any element $x$ in the space $|f(x)| \leq\|f\| \cdot d(x, \operatorname{Ker}(f))$. Applying this fact to the Banach space $M$ and to the linear functional $\phi-\delta$ on $M$, for any $G \in \overline{F\left(\mathcal{R}^{*}(K)\right)}$ we get

$$
\left\|x_{1}\left(G x_{0}^{*}\right)\right\|=|\delta(G)|=|(\phi-\delta)(G)| \leq\|\phi-\delta\|_{M^{*}} \cdot d(G, M \cap \operatorname{Ker}(\phi-\delta)),
$$

where $\|\phi-\delta\|_{M^{*}}$ is the norm of $\phi-\delta$ in $M^{*}$. Since, $C\left(K, X^{*}\right) \subset M \cap \operatorname{Ker}(\delta-\phi)$ we have $d(G, M \cap \operatorname{Ker}(\delta-\phi)) \leq\||| G \mid\|$. Let $k=\|\delta-\phi\|_{M^{*}}$ and $x_{0}^{* *}=\frac{1}{k} \cdot x_{1}$. Then $\left|x_{0}^{* *}\left(G x_{0}^{*}\right)\right| \leq|||G|||$.

Definition 1. A nonzero element $x_{0}^{*} \in X^{*}$ is said to be a quasinoncyclic vector for an algebra $\mathcal{R}^{*}$ of $L\left(X^{*}\right)$ if there is a weak ${ }^{*}$ compact set $K$ containing $x_{0}^{*}$ and a nonzero element $x_{0}^{* *}$ in $X^{* *}$ such that the following inequality holds for all elements $A \in \mathcal{R}^{*}$

$$
\left|x_{0}^{* *}\left(A x_{0}^{*}\right)\right| \leq\left.|||A|\right|_{K} .
$$

The element $x_{0}^{*}$ is said to be a quasinoncyclic vector for $\overline{F\left(\mathcal{R}^{*}(K)\right)}$ if the following inequality holds for all elements $G \in \overline{F\left(\mathcal{R}^{*}(K)\right)}$

$$
\left|x_{0}^{* *}\left(G x_{0}^{*}\right)\right| \leq|||G| \|_{K} .
$$

Corollary 1. Either $\mathcal{R}$ has nontrivial invariant subspaces or any closed ball $K$ of positive radius in $X^{*}$ contains a dense set of quasinoncyclic vectors for $\overline{F\left(\mathcal{R}^{*}(K)\right)}$. 
Proof. Suppose $\mathcal{R}$ has no nontrivial invariant subspaces. Let $K_{0}$ be any closed ball of arbitrary small positive radius contained in $K$. Then by Theorem 1 , there are nonzero elements $x_{0}^{*} \in K_{0}$ and $x_{0}^{* *} \in X^{* *}$ such that

$$
\left|x_{0}^{* *}\left(G x_{0}^{*}\right)\right| \leq \||| G||_{K_{0}}
$$

for all $G \in \overline{F\left(\mathcal{R}^{*}\left(K_{0}\right)\right)}$. Since for any $G \in \overline{F\left(\mathcal{R}^{*}(K)\right)},\left.G\right|_{K_{0}} \in \overline{F\left(\mathcal{R}^{*}\left(K_{0}\right)\right)}$ and $\left\|\left.\left|G_{K_{0}}\right|\right|_{K_{0}} \leq\right\||| G \mid \|_{K}$, the proof follows.

The next definition generalizes the PS property of [L2].

Definition 2. We will say that a subalgebra $\mathcal{R}$ of $L(X)$ has the Lomonosov-PearcySalinas (LPS) property if there is a weak-star compact subset $K$ of $X^{*}$, a quasinoncyclic vector $x_{0}$ for $\overline{F\left(\mathcal{R}^{*}(K)\right)}$, and a net $\left(G_{\alpha}\right)$ in $\overline{F\left(\mathcal{R}^{*}(K)\right)}$, such that $\left\|\mid G_{\alpha}\right\| \| \rightarrow 0$ and $G_{\alpha}\left(x_{0}\right) \rightarrow z_{0}$ for some $z_{0} \neq 0$.

Remark 1 . For any weakly closed proper subalgebra $\mathcal{R}$ of $L(X)$ with the LPS property, $\mathcal{R}^{*}$ has nontrivial invariant subspaces.

Proof. Let $x_{0}, K,\left(G_{\alpha}\right)$, and $z_{0}$ be as in the definition of LPS. By the hypothesis, there is a vector $x_{0}^{*}$ in $X^{* *}$ such that $\left|x_{0}^{*}\left(G x_{0}\right)\right| \leq\||| G \mid\|_{K}$ for all $G \in \overline{F\left(\mathcal{R}^{*}(K)\right)}$. It follows that $\left|x_{0}^{*}\left(A^{*} G_{\alpha} x_{0}\right)\right| \leq\|\| A^{*} G_{\alpha}\|\mid \leq\| A^{*}\|\cdot\| G_{\alpha}\|\| \rightarrow 0$. So, $x_{0}^{*}\left(A^{*} z_{0}\right)=0$ for all $A^{*} \in \mathcal{R}^{*}$.

Note 1. Recall [I] p. 174] that if $X^{*}$ is separable, then $X$ can be renormed so that every boundary point of any closed ball $K$ in $X^{*}$ relative to the new dual norm is a $w^{*}$-to-norm continuity point of the ball. That is, if $x^{*}$ is in the boundary of $K$ and $\left(x_{n}^{*}\right)$ is a sequence in $K$ converging $w^{*}$ to $x^{*}$, then $\left(x_{n}^{*}\right)$ converges in norm to $x^{*}$. The $l^{p}$ space norm has this property for $1<p<\infty$. Note also that a point $y$ of $X^{*}$ is a noncylic vector for an algebra of operators on $X^{*}$ iff it is so when $X^{*}$ is considered with any equivalent norm.

Proposition 1. Suppose $X^{*}$ is separable. Suppose that $X$ has been renormed so that every boundary point of any closed ball in $X^{*}$ relative to the new dual norm is a $w^{*}$-norm continuity point of the ball. Then, for any weakly closed proper subalgebra $\mathcal{R}$ of $L(X)$ with $\operatorname{Ker}\left(\mathcal{R}^{*}\right)=0$ the following are equivalent.

(1) There is a closed ball $K$ of positive radius, a nonzero point $x_{0}^{*}$ on the boundary of $K$, and a nonzero point $x_{0}^{* *} \in X^{* *}$ such that $\left|x_{0}^{* *}\left(G x_{0}^{*}\right)\right| \leq\||| G \mid\|$ for all $G \in \overline{F\left(\mathcal{R}^{*}(K)\right)}$.

(2) $\mathcal{R}$ has the LPS property.

(3) $\mathcal{R}^{*}$ has nontrivial invariant subspaces.

Proof. (1) $\Longrightarrow(2)$. Suppose there are points $x_{0}^{* *}$ and $x_{0}^{*}$ as in (1). Since $K$ is $w^{*}$-separable there is a metric $d$ on $K$ which gives the $w^{*}$ topology on $K$. By the hypothesis on the norm of $X$ the point $x_{0}^{*}$ is a point of $w^{*}$-norm continuity. So, there exist strictly decreasing sequences $\left(\epsilon_{n}\right)$ and $\left(\delta_{n}\right)$ of positive real numbers converging to zero such that for any $x^{*} \in K$, if $d\left(x^{*}, x_{0}^{*}\right)<\delta_{n}$, then $\left\|x^{*}-x_{0}^{*}\right\|<\epsilon_{n}$. Let $U_{n}=\left\{x^{*} \in K: d\left(x^{*}, x_{0}^{*}\right)<\delta_{n}\right\}$. Then, $U_{n-1}^{c} \cap{\overline{U_{n}}}^{*}=\emptyset$. By normality of the metric there is a $w^{*}$ continuous function $f_{n}: K \rightarrow[0,1]$ such that $f_{n}\left(U_{n-1}^{c}\right)=0$ and $f_{n}\left({\overline{U_{n}}}^{w^{*}}\right)=1$. Let $A_{0}^{*} \in \mathcal{R}^{*}$ be any element such that $A_{0}^{*} x_{0}^{*} \neq 0$. Then, $f_{n} A_{0}^{*} \in \overline{F\left(\mathcal{R}^{*}(K)\right)}$ and $\left(f_{n} A_{0}^{*}\right) x_{0}^{*} \rightarrow A_{0}^{*} x_{0}^{*}$ (actually, $\left(f_{n} A_{0}^{*}\right)\left(x_{0}^{*}\right)=f_{n}\left(x_{0}^{*}\right) A_{0}^{*}\left(x_{0}^{*}\right)=$ $\left.A_{0}^{*}\left(x_{0}^{*}\right)\right)$. Define $K_{n}(y)=f_{n}(y) A_{0}^{*} x_{0}^{*} \forall y \in K$ and $\forall n$. Then, $K_{n} \in C\left(K, X^{*}\right)$. 
Claim: $\left\|f_{n} A_{0}^{*}-K_{n}\right\| \rightarrow 0$.

Proof. $\left\|f_{n} A_{0}^{*}(y)-K_{n}(y)\right\|=\left\|f_{n}(y) A_{0}^{*}(y)-f_{n}(y) A_{0}^{*} x_{0}^{*}\right\|$ for all $y \in K$. For $y \in$ $U_{n-1}^{c},\left\|f_{n}(y) A_{0}^{*}(y)-f_{n}(y) A_{0}^{*} x_{0}^{*}\right\|=0$. For $y \in U_{n-1},\left\|f_{n}(y) A_{0}^{*}(y)-f_{n}(y) A_{0}^{*} x_{0}^{*}\right\|=$ $\left|f_{n}(y)\right|\left\|A_{0}^{*}(y)-A_{0}^{*} x_{0}^{*}\right\| \leq\left\|A_{0}^{*}(y)-A_{0}^{*} x_{0}^{*}\right\| \leq\left\|A_{0}^{*}\right\| \cdot\left\|y-x_{0}\right\|<\epsilon_{n-1} \rightarrow 0$.

By the claim $\left\||| f_{n} A_{0}^{*} \mid\right\| \rightarrow 0$. This proves (2) with $G_{n}=f_{n} A_{0}^{*}$, and $z_{0}=A_{0}^{*} x_{0}^{*}$.

$(2) \Longrightarrow(3)$. Follows from Remark 1.

$(3) \Longrightarrow(1)$. This follows from the fact that $y^{* *}\left(A^{*}\left(y^{*}\right)\right)=0$ for all $A^{*} \in$ $\overline{F\left(\mathcal{R}^{*}(K)\right)}$ iff $y^{* *}\left(G y^{*}\right)=0$ for all $G \in \overline{F\left(\mathcal{R}^{*}(K)\right)}$.

Suppose $B$ is reflexive. Suppose $K$ is the unit ball of $B$. Then, for each extreme point $\phi$ of the unit ball of $L(B)^{*}$, there are nets $\left(x_{\alpha}\right)$ and $\left(x_{\alpha}^{*}\right)$ in the unit balls of $B$ and $B^{*}$ such that $\phi(T)=\lim x_{\alpha}^{*}\left(T x_{\alpha}\right)$ for all $T \in L(B)$. We don't know the original reference of this result. However, this follows from the proof of Theorem 1 of [Rao]. If $\phi$ has this form, we shall write $\phi=\left(x_{\alpha} \otimes x_{\alpha}^{*}\right)_{\alpha}$.

For a class of reflexive Banach spaces $X$ all the points of the form $x \otimes x^{*}$ with $\|x\|=\left\|x^{*}\right\|=1, x \in X$, and $x^{*} \in X^{*}$ are extreme points of the unit ball in the predual $X \otimes_{\pi} X^{*}$ as well as of the unit ball in the dual $L(X)^{*}$ of $L(X)$. The invariant subspace problem for a weakly closed subalgebra of $L(X)$, for a reflexive Banach space $X$, asks if there is an extreme point of the form $x \otimes x^{*}$ of the unit ball in the predual of $L(X)$ which annihilates the subalgebra. So it is natural to pose a weaker problem as follows.

Problem. Suppose $X$ is a reflexive Banach space for which all the points of unit norm of the form $x \otimes x^{*}$ are extreme points of the unit ball in the predual $X \otimes_{\pi} X^{*}$ as well as of the unit ball in the dual of $L(X)$. Suppose $\mathcal{R}$ is a proper weakly closed subalgebra of $L(X)$. Is there an extreme point $\phi=\left(x_{\alpha} \otimes x_{\alpha}^{*}\right)_{\alpha}$ of the unit ball of $L(X)^{*}$ with $\left\|x_{\alpha}\right\| \leq 1,\left\|x_{\alpha}^{*}\right\| \leq 1, x_{\alpha} \rightarrow x_{0} \neq 0$ weakly, $x_{\alpha}^{*} \rightarrow x_{0}^{*} \neq 0$ weakly, and $\lim \left(x_{\alpha}^{*}\left(A x_{\alpha}\right)\right)=0$ for all $A \in \mathcal{R}$ ? We can ask the same question relaxing the condition on $\phi$ to be an extreme point, but keeping the condition that $\left\|x_{\alpha}\right\| \leq\|\phi\|$ and $\left\|x_{a}^{*}\right\| \leq\|\phi\|$.

Recall [G] that a result of J. Glimm states that if a linear functional $\phi$ annihilates all the compact operators in $L(H)$, where $H$ is the Hilbert space, then there is a bounded net $\left(x_{\alpha}\right)$ and a bounded net $\left(x_{\alpha}^{*}\right)$ such that $x_{\alpha} \rightarrow 0$ weakly and $x_{\alpha}^{*} \rightarrow 0$ weakly and $\phi=\left(x_{\alpha} \otimes x_{\alpha}^{*}\right)_{\alpha}$. D. Hadwin [H1] defined a Banach space $Y$ to be a Glimm space if for every $\phi \in L(Y)^{*}$ which annihilates the set of all compact operators in $L(Y)$, there are bounded nets $\left(y_{\alpha}\right)$ and $\left(y_{\alpha}^{*}\right)$ in $Y$ and $Y^{*}$ such that $y_{\alpha} \rightarrow 0$ weakly, $y_{\alpha}^{*} \rightarrow 0$ weak-star and $\phi=\left(y_{\alpha} \otimes y_{\alpha}^{*}\right)_{\alpha}$. He proved that a class of spaces including all the $l^{p}$ spaces for $1 \leq p<\infty$ are Glimm spaces. (We refer the readers to [H1] for the exact formulation and for some other very interesting results.) Employing Glimm's result, V. Lomonosov proved Theorem 2 of [L2] which is for a Hilbert space. We shall present a generalization of this result for Glimm spaces. The proof is similar to that of Theorem 2 of [L2]. It can also be obtained from Theorem 1 of this paper.

Corollary 2. Suppose $X$ is a reflexive Glimm space. Suppose $\mathcal{R}$ is a proper weakly closed subalgebra of $L(X)$. Then there are bounded nets $\left(x_{\alpha}^{*}\right)$ and $\left(x_{\alpha}\right)$ in $X^{*}$ and $X$ with $x_{\alpha}^{*} \rightarrow x_{0}^{*} \neq 0$ weakly and $x_{\alpha} \rightarrow x_{0} \neq 0$ weakly such that for every $A \in \mathcal{R}$

$$
x_{\alpha}^{*}\left(A x_{\alpha}\right) \rightarrow 0 .
$$


Note 2. Let $\left(x_{\alpha}\right)$ and $\left(x_{\alpha}^{*}\right)$ be the nets in Corollary 2 .

(1) If $K$ is any weakly compact set containing the net $\left(x_{\alpha}^{*}\right)$, then the conclusion of this corollary implies that for all $G \in \overline{F\left(\mathcal{R}^{*}(K)\right)}$,

$$
x_{\alpha}\left(G x_{\alpha}^{*}\right) \rightarrow 0 .
$$

(2) The functional $\phi \in L(X)^{*}$ defined by $\phi(A)=\lim x_{\alpha}^{*}\left(A x_{\alpha}\right)$ is not necessarily an extreme point of the unit ball of the dual space even when $\left\|x_{\alpha}^{*}\right\| \leq 1$ and $\left\|x_{\alpha}\right\| \leq 1$.

I am very pleased to thank Paul Bourdon and Victor Lomonosov for their suggestions and criticisms that improved the presentation of this article.

\section{REFERENCES}

[G] James Glimm, A Stone-Weierstrass theorem for $C^{*}$-algebras, Ann. of Math. (2) 721960 216-244. MR 22:7005

[H1] Don Hadwin, A reflexivity theorem for subspaces of Calkin algebras, J. Funct. Anal. 123 (1994), no. 1, 1-11. MR 96b:47054

[H2] Don Hadwin, An operator still not satisfying Lomonosov's hypothesis, Proc. Amer. Math. Soc. 123 (1995), no. 10, 3039-3041. MR 95m:47006

[I] Vasile I. Istratescu, Strict convexity and complex strict convexity. Theory and applications, Lecture Notes in Pure and Applied Mathematics, 89. Marcel Dekker, Inc., New York, 1984. MR 86b:46023

[L1] Victor Lomonosov, Invariant subspaces of the family of operators that commute with a completely continuous operator, (Russian) Funkcional. Anal. i Priložen. 7 (1973), no. 3, 55-56. MR 54:8319

[L2] Victor Lomonosov, An Extension of Burnside's Theorem to Infinite Dimensional Spaces, Israel Journal of Mathematics, 75, (1991) 329-339. MR 93h:47007

[Rao] T.S.S.R.K. Rao, A note on extreme points of $W C(K, W)^{*}$, J. Ramanujan Math. Soc. Vol. 9, No. 2 (1994) 215-219. MR 95j:46039

Department of Mathematics and Statistics, Mississippi State University, P.O. Box MA, Mississippi State, Mississippi 39762

E-mail address: shmansr@aol.com 